\title{
The interaction of long and compressible piles with the surrounding and underlying subgrade soils
}

\author{
Zaven Ter-Martirosyan ${ }^{1, *}$, Ivan Luzin $^{1}$ and Mukim Yakubov $^{2}$ \\ ${ }^{1}$ Moscow State University of Civil Engineering, Yaroslavskoe shosse, 26, Moscow, 129337, Russia \\ ${ }^{2}$ Samarkand State Architectural Construction Institute, Department of car roads, soil bases and \\ foundations, 140147 Samarkand, Uzbekistan
}

\begin{abstract}
The article specifies the formulation and solution of problems on the interaction of a long incompressible and compressible pile with the surrounding and underlying subgrade firm soil by an analytical method. It is indicated, that there is the significant effect of the compressibility of the pile shaft on the patterns of force distribution applied to the long pile top between the side surface of the pile and its lower end. The force that comes on the pile toe bulb substantially depends on the ratio of the stiffness of the surrounding and underlying soils $\left(G_{1} / G_{2}\right)$. With the decrease of this ratio, all things being equal, the force on the pile toe bulb increases. It grows with a decrease in the ratio of the radii of the pile and the surrounding thick-wall soil cylinder $(G / a)$. The analysis of the problem solution showed that it is possible to find the optimal ratios between the parameters $G_{1} / G_{2}, b / a, l / a$, at which the force on the pile toe bulb reaches the optimum value wherein the bearing capacity of the underlying soil is at the most used at a given degree of approximation to the limit value.
\end{abstract}

\section{Introduction}

Long bored piles are now widely used in construction under difficult engineering and geological conditions. The length of such piles reaches $100 \mathrm{~m}$, and the diameter is 2.5-3.0 $\mathrm{m}$, and their bearing capacity reaches several thousand tons. Full-scale experiments are carried out to quantify the interaction of such piles with the surrounding and underlying soils and determine their bearing capacity and sediments $[1,2]$. However, they are very expensive. Therefore, analytical and numerical decisions on the interaction of a long pile with the surrounding soils remain relevant. Analytical solution of the problem to solve allows finding the optimal length and diameter of the pile, at which the force on pile toe bulb will be optimal for a given degree of approximation to the limiting state. Existing analytical solutions of this problem do not take into account the compressibility of the pile shaft, which is reflected in the patterns of distribution, force on the pile top between its

\footnotetext{
* Corresponding author: gic-mgsu@ mail.ru
} 
lateral surface and its lower end. This article presents the formulation and solution of problems on the interaction of long and compressible piles with surrounding and underlying soils, including the definition of settlement and bearing capacity of the pile, as well as the distribution of force on the pile head between its side surface and under pile toe bulb. They are necessary to determine the number and pitch of piles under the piled rafts with high grillage at (5-6) $d$ pitch.

Experimental studies show that the force coming to the lower end of the pile does not exceed $15-20 \%$; therefore the main part of the pile comes from the side surface of it. This is confirmed by the results of full-scale experiments (Table 1) and numerical calculations of the mode of deformation of pile and the surrounding soil during their interactions (Fig. 1). The analysis of these curves and the calculations of other authors [1] show that the interaction of long piles with the surrounding and underlying soils involves a limited amount of soil both around it and under its lower end. This makes it possible to consider a thick-wall soil cylinder of limited dimensions (diameter, length) as a calculated geomechanical model, containing a long pile and represented by relatively dense soils, and there is practically no influence of the pile outside this volume.

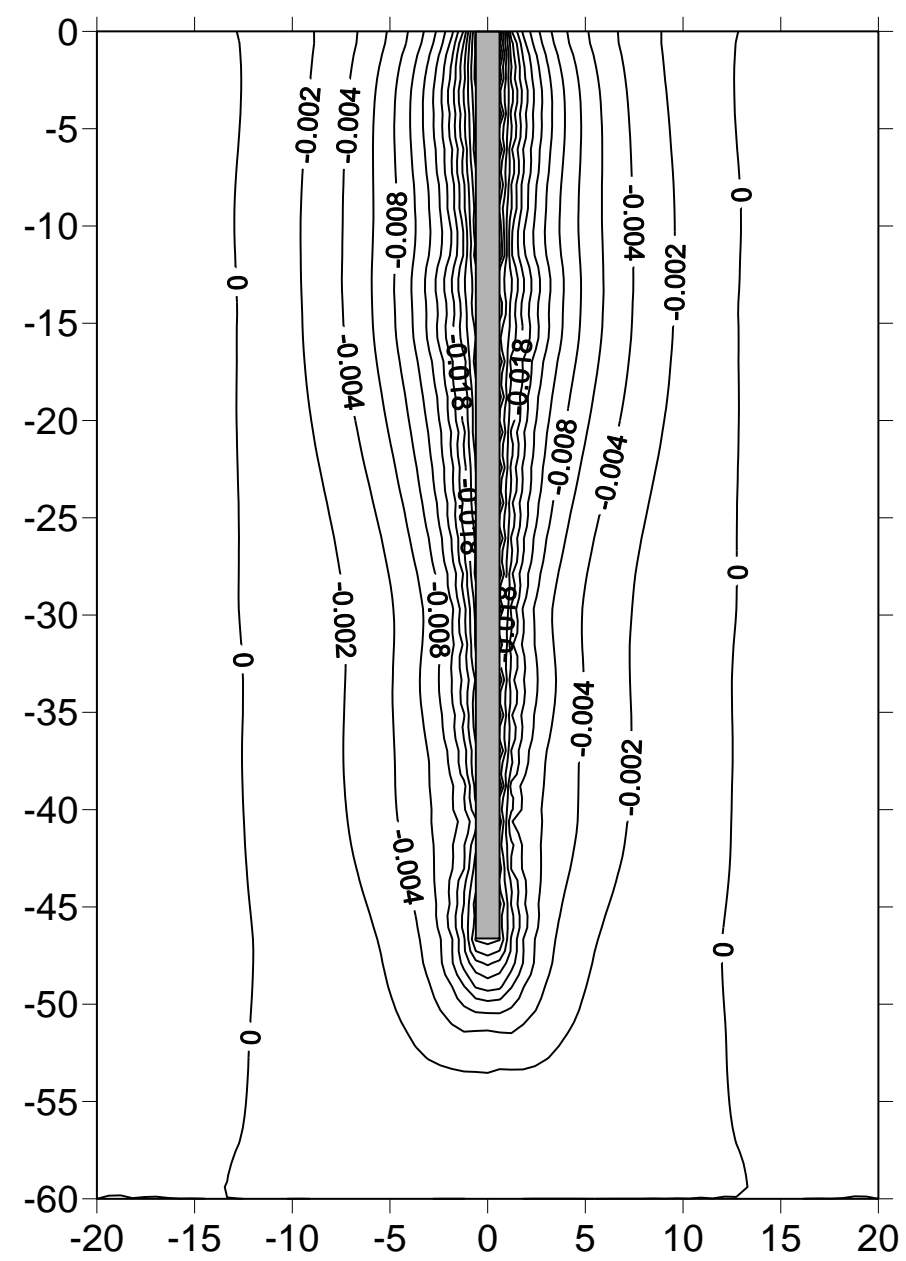

Fig. 1. Isofields of vertical displacement of soil around a pile $46.8 \mathrm{~m}$ long and $1.2 \mathrm{~m}$ in diameter (calculated by FEM) 
Below is given the formulation and analytical solution of problems on the interaction of long, incompressible and compressible bored piles with the surrounding and underlying linearly deformable soils, taking into account their linear properties under the action of constant force acting on the pile top.

Table 1.1. Force (t) on a pile $46.8 \mathrm{~m}$ long according to test results

\begin{tabular}{|c|c|c|c|c|c|c|}
\hline \multicolumn{7}{|c|}{ Distance from the pile top, $m$} \\
\hline \multirow{14}{*}{ 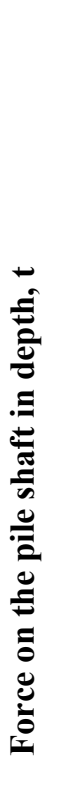 } & $0 \mathrm{~m}$ & $2 \mathrm{~m}$ & $10 \mathrm{~m}$ & $17 \mathrm{~m}$ & $39 \mathrm{~m}$ & $45 \mathrm{~m}$ \\
\hline & 250 & 250 & 246 & 195 & 18 & - \\
\hline & 500 & 500 & 456 & 362 & 75 & 14 \\
\hline & 750 & 750 & 690 & 580 & 178 & 50 \\
\hline & 1000 & 1000 & 919 & 818 & 309 & 93 \\
\hline & 1250 & 1250 & 1174 & 1054 & 456 & 144 \\
\hline & 1500 & 1500 & 1376 & 1262 & 601 & 205 \\
\hline & 1750 & 1750 & 1651 & 1523 & 769 & 292 \\
\hline & 2000 & 2000 & 1906 & 1758 & 945 & 386 \\
\hline & 2100 & 2100 & 2086 & 1949 & 1045 & 457 \\
\hline & 2200 & 2200 & 2138 & 2001 & 1083 & 477 \\
\hline & 2300 & 2300 & 2234 & 2084 & 1143 & 518 \\
\hline & 2400 & 2400 & 2334 & 2177 & 1414 & 561 \\
\hline & 2500 & 2500 & 2428 & 2268 & 1264 & 587 \\
\hline
\end{tabular}

\section{The interaction of a single long incompressible pile with the surrounding and underlying soils}

As a computational geomechanical model, we consider a thick-walled soil cylinder of limited size $\left(l_{1}, 2 a\right)$, containing a long pile, and an underlying relatively dense soil $\left(G_{2}>G_{1}\right)$, assuming that the lower end of the pile is slightly $(\subset l)$ buried in the underlying layer of dense soil, i.e. ${ }_{-}^{-} l_{-}^{-} l$ (Fig.2.1.) 


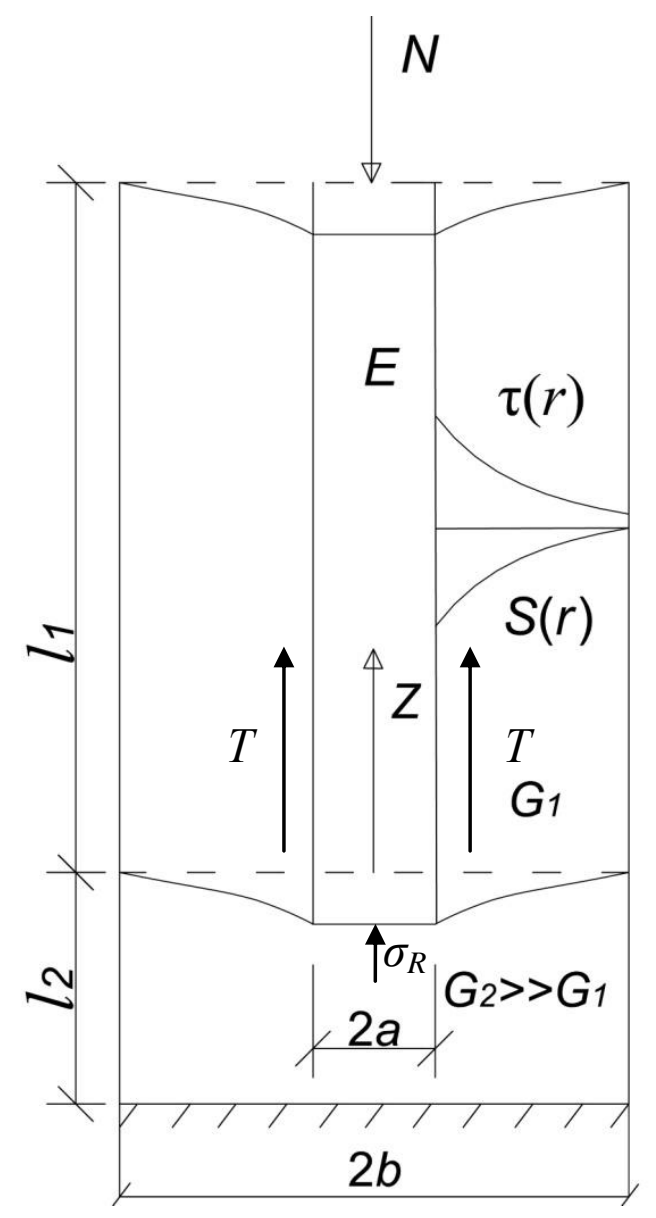

Fig. 2. The design model of the interaction of a long pile with the surrounding and underlying soils

Problem solution

The pile settlement due to tangential stresses around it can be determined through angular deformations by the type dependence

$$
S=\int \gamma(r) d r+C
$$

where $C$ - integration constant determined from boundary conditions, and

$$
\gamma(r)=\tau_{a}\left(a / r \cdot G_{1}\right) ; \tau_{a}=T / 2 \pi a l_{1}
$$

Substituting these values into (2.1), we obtain

$$
S(r)=\frac{\tau_{a} a}{G_{1}} \int \frac{d r}{r}+C=-\frac{\tau_{a} a}{G_{1}} \ln (r)+C
$$

while $r=b, S(r)=0 \rightarrow C=\frac{\tau_{a} a}{G_{1}} \ln b$,

And finally

$$
S(r)=\frac{\tau_{a}(r)}{G_{1}} \ln \frac{b}{r}
$$


While $r=a$ we obtain the maximum value of pile settlement from the action of tangential stresses, i.e. we obtain

$$
S(a)=\frac{\tau_{a} a}{G_{1}} \ln \frac{b}{a}
$$

The settlement of the pile at its lower end can be determined in accordance with the known formula for determining the precipitation of a round rigid stamp [3,4]

$$
S_{R}=\frac{R\left(1-v_{2}\right)}{4 G_{2} a} K(l)=\frac{\pi a \sigma_{R}\left(1-v_{2}\right)}{4 G_{2}} K(l)
$$

where $K(l)<1$ - coefficient taking into account the depth of the lower end of the pile.

Comparing (2.5) and (2.6) we obtain

$$
\tau_{a}=\frac{G_{1}}{G_{2}} \frac{\pi \sigma_{R}\left(1-\gamma_{r}\right)}{4 \ln (b / a)} K(l)=\text { const }
$$

From the equilibrium condition of the pile, it follows that

$$
\pi a^{2} \cdot \sigma_{N}=\pi a^{2} \cdot \sigma_{R}+2 \pi a l \cdot \tau_{a}
$$

Substituting the value from (2.7) here, we obtain:

$$
\sigma_{N}=\sigma_{n}+\frac{l}{a} \frac{G_{1}}{G_{2}} \frac{\pi \sigma_{R}\left(1-v_{2}\right) K(l)}{2 \ln (b / a)}
$$

And finally

$$
\sigma_{R}=\sigma_{N} / A_{1}
$$

where

$$
A_{1}=1+\frac{l}{a} \frac{G_{1}}{G_{2}} \frac{\pi\left(1-v_{2}\right) K(l)}{2 \ln (b / a)}
$$

Substituting the value $\sigma_{R}$ from (2.7), we obtain

$$
\tau_{a}=\frac{G_{1}}{G_{2}} \frac{\pi\left(1-v_{2}\right) K(l)}{4 \ln (b / a)}\left(\sigma_{N} / A_{1}\right)=\sigma_{N}\left(B_{1} / A_{1}\right)
$$

where $B_{1}=\frac{G_{1}}{G_{2}} \frac{\pi\left(1-v_{2}\right) K(l)}{4 \ln (b / a)}$.

The pile draft is determined by (2.5) or (2.6), substituting the expressions $\sigma_{R}$ from (2.10) or $\tau_{a}$ from (2.11) into them.

\section{The interaction of long compressible piles with the surrounding and underlying soils}

From consideration of the equilibrium condition of an elementary pile length $d z$, it follows that

$$
\frac{\partial \sigma_{z}}{\partial z}=\frac{z}{a} \tau_{z} ; \rightarrow \frac{d \sigma_{z}}{d z}=\frac{r}{a} \cdot \frac{S \cdot G_{1}}{a \cdot \ln (b / a)}
$$

Considering that $\sigma_{z}=\varepsilon_{z} E=(d s / d z) E$, we obtain

It follows that

$$
\frac{d s}{d z}=\frac{\sigma z}{E} \rightarrow \frac{d^{2} \sigma_{z}}{d z^{2}}=\frac{d \sigma_{z}}{d z} \frac{1}{E}=\frac{r}{a^{2}} \frac{S G_{1}}{E \ln (l / a)}
$$




$$
\frac{d^{2} S}{d z^{2}}-\lambda^{2} S=0
$$

where

$$
\lambda^{2}=\frac{2 G_{1}}{a^{2} E \ln (b / a)}
$$

Solution (3.4) is known [5-7] and has the form:

$$
S(z)=C_{1} \operatorname{sh} \lambda z+C_{2} \operatorname{ch} \lambda z
$$

moreover

$$
d S / d z=\mathrm{C}_{1} \lambda \operatorname{sh} \lambda z+\mathrm{C}_{2} \lambda \operatorname{ch} \lambda z
$$

It follows from the boundary conditions that

$$
z=0 \rightarrow S=S_{0} \text { and } z=l \rightarrow \frac{d s}{d z}=\varepsilon_{z}=\frac{\sigma_{N}}{E}
$$

As with the values $\lambda z=0 \operatorname{sh} 0=0, \operatorname{ch} 0=1$, so $C_{2}=S$, and

$$
\frac{\sigma_{N}}{E}=C_{1} \lambda \operatorname{ch} \lambda l+C_{2} \lambda \operatorname{sh} \lambda l \rightarrow C_{1}=\left(\frac{\sigma_{N}}{\lambda E}-C_{2} \operatorname{sh} \lambda l\right) \frac{1}{\operatorname{ch} \lambda l}
$$

And finally

$$
S(z)=\left(\frac{\sigma_{N}}{\lambda E}-S_{0} \operatorname{sh} \lambda l\right) \frac{\operatorname{sh} \lambda z}{\operatorname{ch} \lambda l}+S_{0} \operatorname{ch} \lambda z
$$

Considering that $S(z)=f\left(\tau_{z}\right)$ acc. to $(2.5)$ we can write

$$
\left(\frac{\sigma_{N}}{\lambda E}-S_{0} \operatorname{sh} \lambda l\right) \frac{\operatorname{sh} \lambda z}{\operatorname{ch} \lambda l}+S_{0} \operatorname{ch} \lambda z=\tau(z) \frac{a}{G_{1}} \ln (b / a)
$$

It follows that

$$
\tau(z)=A_{3} \operatorname{sh} \lambda z+B_{3} \operatorname{ch} \lambda z
$$

where

$$
A_{3}=\left(\frac{\sigma_{N}}{\lambda E}-S_{0} \operatorname{sh} \lambda l\right) \frac{G_{1}}{a \ln (b / a)} ; \quad B_{3}=\frac{S_{0} G_{1}}{a \ln (b / a)}
$$

from (3.10) it follows that $S(l)>\mathrm{S}_{0}$. This corresponds to the conditions of compressibility of the pile shaft. We define the unknown $S_{0}$ as follows.

The total force due to tangential stresses $T$ can be determined by integrating $\tau(z)$ from 0 to $l$. Then we obtain

$$
T=A_{3} \frac{\operatorname{ch} \lambda l-1}{\lambda}+B_{3} \frac{\operatorname{sh} \lambda l}{\lambda}
$$

From the equilibrium condition it follows that

$$
T=\pi a^{2}\left(\sigma_{N}-\sigma_{R}\right)
$$

Substituting the value $A_{3}$ and $B_{3}$ from (3.12) into equation (2.13) and equating the obtained expression with (3.14) we obtain

$$
\left(\frac{\sigma_{N}}{\lambda E}-S_{0} \operatorname{sh} \lambda l\right) \frac{G_{1}}{a \ln (b / a)} \frac{\operatorname{ch} \lambda l-1}{\lambda}+\frac{S_{0} G_{1}}{a \ln (b / a)} \frac{\operatorname{sh} \lambda l}{\lambda}=\pi a^{2}\left(\sigma_{N}-\sigma_{R}\right)
$$

Given that $S_{0}$ is determined by (2.6) we obtain:

$$
\sigma_{N}\left(\frac{G_{1}}{\lambda E} \frac{\operatorname{ch} \lambda l-1}{a \ln (b / a)}-\pi a^{2}\right)=\sigma_{R}\left[\frac{\pi a\left(1-v_{2}\right)}{4 G_{2} \lambda} \frac{G_{1}}{\ln (b / a)}(\operatorname{ch} \lambda l-1-\operatorname{sh} \lambda l)-\pi a^{2}\right]
$$

It follows that 


$$
\sigma_{R}=\sigma_{N} D / E
$$

where $D$ and $E$ determined by expressions of the form:

$$
E=\frac{\pi a\left(1-v_{2}\right)}{4 G_{2} \lambda} \frac{G_{1}}{\ln (b / a)}(\operatorname{ch} \lambda l-1-\operatorname{sh} \lambda l)-\pi a^{2} ; \quad D=\frac{G_{1}}{\lambda E} \frac{\operatorname{ch} \lambda l-1}{a \ln (b / a)}-\pi a^{2}
$$

The expression $\tau(z)$ can be defined by substituting into (3.11) $S_{0}$ with (2.6) taking into account (3.12).

Accordingly, $\sigma(z)$ can be determined from the resulting expression, based on condition (3.12) by integrating of $\tau(z)$ :

$$
\sigma(z)=\frac{2}{a} \int \tau(z) d z+C
$$

where $C$ is determined from the condition when $z=0 \rightarrow \sigma(z=0)=\sigma_{R}$, so we obtain

$$
\sigma(z)=\frac{2}{a \lambda}\left(A_{3} \operatorname{sh} \lambda z+B_{3} \operatorname{ch} \lambda t\right)+\sigma_{R}
$$

where $A_{3}$ and $B_{3}$ are still defined by (3.12) in which $S_{0}$ is determined by (2.5).

Finally, to determine the vertical displacement of the pile by $z$, notably $S(z)$, you should substitute the values $C_{1}$ and $C_{2}$ from (3.18) in (3.6).

Thus, the problem is completely solved.

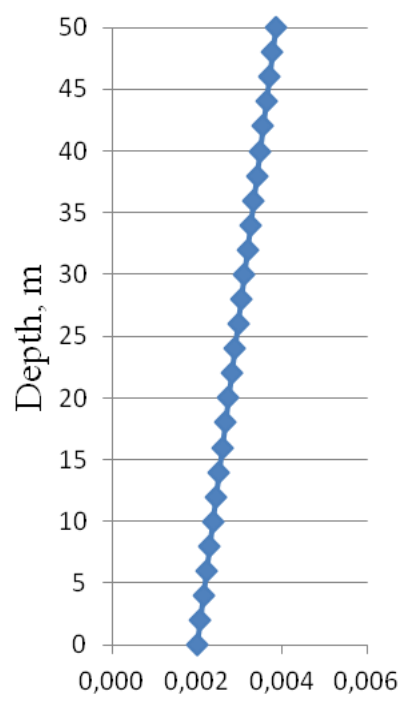

Settlement, $\mathrm{m}$

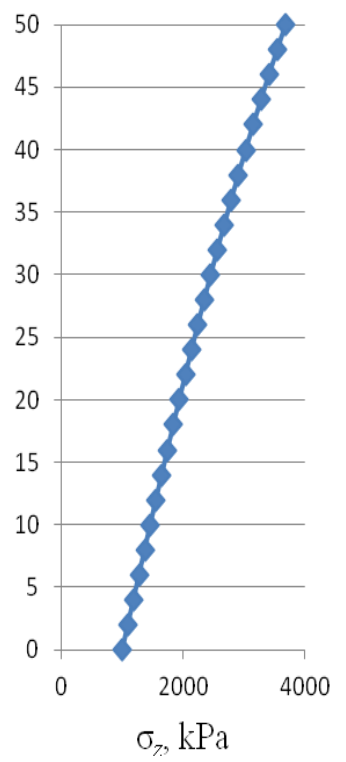

(b)

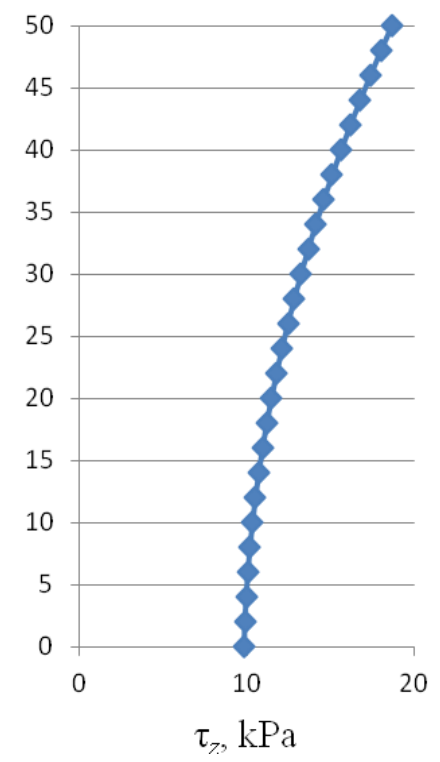

(c)

Fig. 3. Distribution of vertical displacements $S(t)$ (a), vertical stresses $\sigma(z)$ (b) and tangential stresses $\tau(z)$ (c) along the pile, calculated according to formulas (3.5), (3.19) and (3.11)

\section{Conclusion}

1. When a long compressible pile interacts with the surrounding and underlying soils under the influence of a constant load on the pile top, a difficult and non-uniform mode of 
deformation arises in them, which can transform in space with a variable load on the pile top.

2. An analysis of the solution of the problem showed a significant effect of the compressibility of a long pile on the distribution of force on the pile top between the force at the lower end of the pile, the tangential stresses along the side surface of the pile, and the stresses in the pile shaft. With increasing pile length, the force at its lower end decreases to zero.

3. To assess the bearing capacity of soils of the lower layer, it is necessary to compare the force at the lower end of the pile on the limiting state.

4. Solving this problem allows finding optimal pile sizes (length, diameter) under given geotechnical conditions so as to ensure the transfer of maximum force at the bottom end of the pile while ensuring a given degree of approximation to the limiting state.

This work was financially supported by the Ministry of Education and Science (state task \# 7.3225.2017/4.6). All tests were carried out using research equipment of The Head Regional Shared Research Facilities of the Moscow State University of Civil Engineering.

\section{References}

1. Ter-Martirosyan Z., Nguen Z.N., Vestnik grazhdanskikh inzhenerov [Bull. of civ. eng.]. 1(10), 52 (2007)

2. Ter-Martirosyan Z., Soil mechanics (ASV, Moscow, 2009)

3. Trofimov V., Soil science (MSU, Moscow, 2005)

4. Tikhonov A., Samorskii A., Equations of mathematical physics (Nedra, Moscow, 1979)

5. Ter-Martirosyan Z., Rheological soil parameters and foundation bases calculations (Stroiizdat, Moscow, 1990)

6. Tsytovitch N., Ter-Martirosyan Z., Fundamentals of applied geomechanics in civil engineering (Vyshaya shkola, Moscow, 1973) 